\title{
POTENTIAL THEORY FOR INFINITELY DIVISIBLE PROCESSES ON ABELIAN GROUPS 1
}

\author{
BY SIDNEY C. PORT AND CHARLES J. STONE
}

Communicated by Henry P. McKean, Jr., February 24, 1969

Let $\$$ be a 2 nd countable locally compact, noncompact Abelian group, and let $X_{t}$ be an infinitely divisible process (henceforth called an i.d. process) with stationary increments taking values in (\$). The transition functions $P^{t}(x, d y)$ of such a process have the Feller property, so via a fundamental theorem in the theory of Markov processes (see $[1$, p. $44 \mathrm{ff}$.$] ) there is a realization of the process as a standard$ Markov process. Henceforth we shall assume that $X_{t}$ is this version of the process. A point $x \in \mathbb{B}$ is called possible if for each neighborhood $N$ of 0 there is a $t>0$ such that $P^{t}(0, N+x)>0$. The set $\Sigma$ of all possible points is a closed subsemigroup of (5). Except when discussing the renewal theorem we will always assume that $\Sigma=B$. The process is called recurrent if $\int_{0}^{\infty} P^{t}(x, A) d t=\infty$ for all nonempty open sets $A$ and all points $x$. Otherwise the process is called transient. In that case $\int_{0}^{\infty} P^{t}(x, A) d t<\infty$ for all compact sets $A$ and all points $x$. A transient process is called strongly transient if $\int_{0}^{\infty} d t \int_{i}^{\infty} P^{s}(x, A) d s<\infty$ for all compact sets $A$ and all points $x$. Otherwise the transient process is called weakly transient. In that case $\int_{0}^{\infty} d t \int_{t}^{\infty} P^{s}(x, A) d s=\infty$ for all nonempty open sets $A$ and all points $x$. Let $f \geqq 0$ be a continuous function with compact support having integral 1 , and set $r(t)=\int_{t}^{\infty} P^{s} f(0) d s$. Then a transient process is strongly transient if and only if $\int_{0}^{\infty} r(t) d t<\infty$. A process is called nonsingular if for some $t>0$ the distribution of $X_{t}$ has a nonsingular component relative to Haar measure on (\$).

Very briefly, our purpose is to show that results in [3] and [4] for random walks on (\$) go over to i.d. processes on (\$), and this we do rather completely. These results are new even for i.d. processes on Euclidean spaces. Space does not permit a detailed description of all these results (much less their proofs) so we will only sketch what has been done. We point out here, however, that although most of the results on random walks have their counterpart for i.d. processes, the proofs of these results in many cases require significant new ideas. Some of these ideas involve indirect reductions to the discrete time case.

Let $B$ be a relatively compact set, and let $T_{B}=\inf \left\{t \geqq 0: X_{t} \in B\right\}$ denote the first hitting time of $B$. We show that a theory of $\lambda$-capacities can be developed for any i.d. process. If the $\lambda$-capacity $C^{\lambda}(B)=0$ for one $\lambda>0$, then this is true for all $\lambda>0$, and then $P_{x}\left(T_{B}<\infty\right)=0$ a.e. On the other hand, if $C^{\lambda}(B)>0$ for some $\lambda>0$, then this is true

1 The preparation of this paper was supported in part by NSF Grant No. GP-8049. 
for all $\lambda>0$, and then $P_{x}\left(T_{B}<\infty\right)>0$ a.e. (and in the nonsingular case for all $x$ ). The $\lambda$-capacity theory is used to give criteria as to when certain sets have positive $\lambda$-capacity. As an example, we show that a point has positive $\lambda$-capacity if and only if the resolvent of the process has a bounded, strictly positive density.

For transient processes we first show that the renewal theorems given in $\$ 3$ of [4] go over nicely to continuous time. However, most of our efforts in the transient case concern the asymptotic behavior, for large $t$, of the quantities $E_{x}\left[f\left(X_{T_{B}}\right) ; t<T_{B}<\infty\right]$, $E_{x}\left[f\left(X_{R_{B}}-\right) ; R_{B}>t\right]$ (where $R_{B}=\sup \left\{t \geqq 0: X_{t} \in B\right\}$ is the last hitting of $B$ ) as well as the quantity

$$
E_{B}(t ; A)=\int_{\mathcal{B}} P_{x}\left(T_{B} \leqq t ; X_{T_{B}} \in A\right) d x .
$$

The results we obtain for these quantities parallel those given in [3] for random walks.

Let $a$ denote the collection of all relatively compact sets such that $\stackrel{\circ}{B} \neq \varnothing$ and $P_{x}\left(T_{\bar{B}}=T_{\mathscr{B}}\right)=1$ a.e. In Euclidean space sets with nice boundaries such as balls, rectangles etc. are in $a$. Of course if $B \in Q$, then $C^{\lambda}(B)>0$. Let $B^{*}$ denote the collection of all relatively compact sets $B$ such that $C^{\lambda}(B)>0$ if the process is nonsingular and let $B^{*}=a$ in the general case. Also let $\Phi^{*}$ denote the collection of all bounded measurable functions with compact support if the process is nonsingular, and let $\Phi^{*}$ denote the collection of all continuous functions with compact support in the general case. We illustrate our results on transient processes with the following examples.

(A) Let $B$ be any Borel set. Then either (i) $P_{x}\left(T_{B}<\infty\right)=1$ a.e. $x$ (and for all $x$ in the nonsingular case), or (ii)

$$
\lim _{t \rightarrow \infty} P_{x}\left(X_{s} \in B \quad \text { for some } s \geqq t\right)=0 \quad \text { a.e. } x \text {, }
$$

and in that case there is a unique measure $\mu_{B}$ supported on $\bar{B}$ (that is finite if $B$ is relatively compact) such that for any Borel set $A$,

$$
\int_{A} P_{x}\left(\tilde{T}_{B}<\infty\right) d x=\int_{\bar{B}} \mu_{B}(d y) \int_{0}^{\infty} P^{s}(y, A) d s .
$$

(B) Assume $C^{\lambda}(B)>0$. Then for all measurable subsets $A \subset B$,

$$
\lim _{t \rightarrow \infty}\left[E_{B}(t+h ; A)-E_{B}(t ; A)\right]=h \mu_{B}(A) .
$$

Moreover, if the process is strongly transient then

$$
E_{B}(t ; A)-t \mu_{B}(A) \uparrow \int_{B} P_{x}\left(\tilde{T}_{B}<\infty\right) P_{x}\left(X_{T_{B}} \in A\right) d x<\infty,
$$


where $\widetilde{T}_{B}$ is the quantity $T_{B}$ for the process $-X(t)$. On the other hand if the process is weakly transient and nonsingular then

$$
E_{B}(t ; A)-t \mu_{B}(A) \sim \mu_{B}(A) C(B) \int_{0}^{t} r(s) d s .
$$

This result is also valid for general weakly transient processes provided $B \in Q$ and $\mu_{B}(|\partial A|)=0$. These results extend those of Spitzer [5] and Getoor [2].

(C) Let $B \in Q^{*}$ and let $f \in \Phi^{*}$. Assume that for an open set $A, \bar{A}$ compact, $\int_{0}^{\infty} e^{\lambda t} P^{t}(0, A) d t=\infty$ for $\lambda>0$. Then for $B \in B^{*}$ and $f \in \Phi^{*}$,

$$
\int_{\Theta} P^{t}(x, d y) E_{y}\left[f\left(X_{T_{B}}\right) ; T_{B}<\infty\right] \sim r(t) \int_{B} f(y) \mu_{B}(d y), \quad t \rightarrow \infty .
$$

(D) Let $f$ be any bounded measurable function having compact support and let $h$ be any continuous function having compact support. Then for $B$ relatively compact and $C^{\lambda}(B) \geqq 0$

$$
\begin{aligned}
& P_{x}\left\{\lim _{t \rightarrow \infty} \int f(y) E_{y-X_{t}}\right. {\left[h\left(X_{T_{B}}\right) \mid T_{B}<\infty\right] d y } \\
&\left.=\left[\int_{\Theta} f(y) d y\right]\left[\int_{\Theta} h(y) \frac{\mu_{B}(d y)}{\mu_{B}(\bar{B})}\right]\right\}=1 \text { a.e. } x
\end{aligned}
$$

(for all $x$ in the nonsingular case).

In addition to these results we also investigate some problems in the potential theory for transient i.d. processes. Among these is the problem of finding all nonnegative functions invariant for the process killed on $B$, the Dirichlet problem for $B$, and the Poisson problem. This last problem is as follows. Given $\phi$ continuous with compact support, find $f \geqq 0$ such that

$$
\begin{gathered}
\lim _{t \downarrow 0} \frac{P^{t} f(x)-f(x)}{t}=\phi(x), \quad x \in \text { (5); } \\
\sup _{t \leq 1} \sup _{x}\left|\frac{P^{t} f(x)-f(x)}{t}\right|<\infty .
\end{gathered}
$$

For recurrent processes we show that if $C^{\lambda}(B)>0$, then in fact $P_{x}\left(T_{B}<\infty\right)=1$ a.e. (and for all $x$ in the nonsingular case). We then show that if $C^{\lambda}(B)>0$ there is a unique probability measure $\mu_{B}$ supported on $\bar{B}$ such that $E_{B}(t ; A) \sim \mu_{B}(A) E_{B}(t, \bar{B})$, and that if also $C^{\lambda}(D)>0$ then $E_{B}(t ; \bar{B}) \sim E_{D}(t ; \bar{D})$. The measure $\mu_{B}(A)$ turns out to be the limit at $\infty$ of a smoothed version of the hitting measure. We also show that to each compact set $B$ there is associated a unique constant $k(B),-\infty \leqq k(B)<\infty$ such that $k(B)>-\infty$ if and only if the Green's function $G_{B}(x, A)$ defined by 


$$
G_{B}(x ; A)=E_{x} \int_{0}^{T_{B}} 1_{A}\left(X_{t}\right) d t
$$

is locally integrable for all compact sets $A$. If $C^{\lambda}(B)=0$, then $k(B)$ $=-\infty$, and for nonsingular processes the converse is true. (This, then, constitutes a generalization to recurrent nonsingular i.d. processes of Kakutani's test for Brownian motion.) In the general case, however, it can happen that $|B|>0$ (so that $C^{\lambda}(B)>0$ ) but yet $k(B)=-\infty$ ! If $k(B)>-\infty$, then we show that the results given on the asymptotic behavior of $G_{B}(x ; A)$ in [4] for random walks carry over in a smoothed version. The sharper, unsmoothed, versions of these results for $G_{B}(x, A)$ and the hitting distribution are valid for all set in $B^{*}$.

In addition to these results we also show that the recurrent potential theory of functions goes over to the continuous time processes. These potential theoretic results are used to find all the nonnegative invariant functions of the process killed on $B$, and all the nonnegative solutions of the Poisson problem. Finally, we also investigate the asymptotic behavior for large $t$ of $E_{x}\left[f\left(X_{T_{B}}\right) ; T_{B}>t\right]$, of $E_{B}(t ; A)$, and of $E_{B}(t ; \bar{B})-E_{D}(t ; \bar{D})$ and here too the results we obtain parallel those given in [4] for random walks. As an example of these results we mention the following.

If for some $\alpha, 0 \leqq \alpha<1$, and some slowly varying function $H(\cdot)$ there is a function $g$ such that $G^{\lambda} g(0) \sim(1 / \lambda)^{\alpha} H(1 / \lambda), \lambda \downarrow 0$, then for $B, D \in B^{*}$,

$$
E_{B}(t ; \bar{B})-E_{D}(t ; \bar{D}) \sim \frac{[k(B)-k(D)] t^{-2 \alpha+1}}{H(t)^{2} \Gamma(2-2 \alpha)}, \quad t \rightarrow \infty .
$$

A result of this type was first given by Spitzer [5] for planar Brownian motion. However, the results given here are new even for recurrent stable processes.

\section{REFERENCES}

1. R. Blumenthal and R. Getoor, Markov processes and potential theory, Academic Press, New York, 1968.

2. R. Getoor, Some asymptotic formulas involving capacity, Z. Wahrscheinlichkeitstheorie und Verw. Gebiete 4 (1965), 248-259.

3. S. C. Port and C. J. Stone, Hitting times for transient random walks on groups, J. Math. Mech. 17 (1968), 1117-1130.

4. - Potential theory for random walks on abelian groups, Acta Math. 122 (1969), 19-115.

5. F. Spitzer, Electrostatic capacity, heat flow and brownian motion, Z. Wahrscheinlichkeitstheorie und Verw. Gebiete 3 (1964), 110-121.

University of California, los Angeles, California 90024 